\title{
Ceftazidime-Avibactam in Combination with In Vitro Non-susceptible Antimicrobials Versus Ceftazidime- Avibactam in Monotherapy in Critically Ill Patients with Carbapenem-Resistant Klebsiella Pneumoniae Infection: A Retrospective Cohort Study
}

\author{
Guanhao Zheng $\cdot$ Jianxin Zhang $\cdot$ Bei Wang $\cdot$ Jiaqi Cai $\cdot$ \\ Lili Wang $\cdot$ Kaixuan Hou $\cdot$ Yan Zhang $\cdot$ Liang Zhang · \\ Zhitao Yang $\cdot$ Juan He (i) $\cdot$ Xiaolan Bian
}

Received: April 12, 2021 / Accepted: June 10, 2021 / Published online: July 9, 2021

(C) The Author(s) 2021, corrected publication 2021

\section{ABSTRACT}

Background: No clinical study has investigated the use of ceftazidime-avibactam combination schemes with an in vitro non-susceptible antimicrobial that could be superior to ceftazidime-avibactam monotherapy against carbapenem-resistant Klebsiella pneumoniae.

Methods: We performed a retrospective cohort study at two tertiary hospitals in China for

Jianxin Zhang and Bei Wang are co-first author.

\section{G. Zheng · J. He (四)}

Department of Pharmacy, Ruijin Hospital

Affifiliated To Shanghai Jiao Tong University School of Medicine, Shanghai, China

e-mail: hejuanwin@126.com

\section{G. Zheng}

Department of Pharmacy, Shanghai Chest Hospital, Shanghai, China

\section{J. Zhang · Y. Zhang}

Department of Pharmacy, Xing'an League People's

Hospital, Ulanhot, China

B. Wang · L. Zhang $(\bowtie)$

Department of Pharmacy, Huashan Hospital Affiliated To Fudan University, Shanghai, China e-mail: cathyzhangliang@126.com

J. Cai

Department of Clinical Laboratory, Kunshan Hospital Affiliated to Nanjing University of Chinese Medicine, Kunshan, China patients with carbapenem-resistant Klebsiella pneumoniae infection treated with ceftazidimeavibactam for at least $72 \mathrm{~h}$. A Cox proportional hazards regression model was used to evaluate covariates that potentially affected 30-day mortality.

Results: Sixty-two patients were eligible for our study; 41 (66.1\%) received ceftazidime-avibactam combination therapy and 21 (33.9\%) received ceftazidime-avibactam monotherapy. The overall 30-day mortality was 33.9\% (21
L. Wang
Department of Pharmacy, Wuxi Branch of Ruijin Hospital, Wuxi, China
K. Hou
Department of Pharmacy, Huaihe Hospital of Henan University, Kaifeng, China

\section{Z. Yang $(\bowtie)$}
Department of Emergency Intensive Care Unit, Ruijin Hospital Affiliated To Shanghai Jiao Tong University School of Medicine, Shanghai, China e-mail: yangzhitao@hotmail.fr

\section{Bian (ه)}
Department of Pharmacy, Luwan Branch of Ruijin Hospital Affifiliated To Shanghai Jiao Tong University School of Medicine, Shanghai, China e-mail: bx170029@163.com 
patients): $24.4 \%(10 / 41)$ and $47.6 \%(11 / 21)$, $P=0.028$, in combination and monotherapy groups, respectively. Combination therapy was significantly associated with lower 30-day mortality (Hazard ratio, 0.167; 95\% Confidence Interval, $0.060-0.465, P=0.001)$. At the same time, a higher APACHE II score, use of vasoactive drugs and comorbidity of organ transplantation were considered factors that increased mortality. The propensity score showed no significant alterations with other variables after adding it to the final model. In the subgroup analysis, the protective effect was revealed when combined with carbapenems, tigecycline or fosfomycin were applied, and in the following subgroups of patients: with sepsis, with creatinine clearance $>50 \mathrm{~mL} / \mathrm{min}$, stayed in the intensive care unit $\leq 30$ days or underwent mechanical ventilation.

Conclusions: Ceftazidime-avibactam combined with another in vitro non-susceptible antimicrobial, especially carbapenems, fosfomycin and tigecycline, could significantly decrease the 30-day mortality rate for critically ill patients with carbapenem-resistant Klebsiella pneumoniae infection. Further investigation should be carried out to confirm this conclusion and identify autofit antimicrobials in ceftazidime-avibactam combination schemes.

Keywords: Ceftazidime-avibactam;

Carbapenem-resistant Klebsiella pneumoniae; Combination therapy; Critically ill patients; Mortality

\section{Key Summary Points}

1. Ceftazidime-avibactam is a novel antimicrobial agent with clinical efficacy against carbapenem-resistant Klebsiella pneumoniae infection.

2. It is unknown whether using ceftazidime-avibactam combination schemes could have a better clinical effectiveness against carbapenem-resistant Klebsiella pneumoniae than ceftazidimeavibactam monotherapy.
3. Ceftazidime-avibactam combined with another in vitro non-active antimicrobial could significantly lower the mortality risk in critically ill patients with carbapenem-resistant Klebsiella pneumoniae infection.

4. Carbapenems, fosfomycin, and tigecycline could be optional concomitant antimicrobials in combination with ceftazidime-avibactam to treat carbapenem-resistant Klebsiella pneumoniae infection.

\section{DIGITAL FEATURES}

This article is published with digital features, including a summary slide, to facilitate understanding of the article. To view digital features for this article go to https://doi.org/10.6084/ m9.figshare.14749536

\section{INTRODUCTION}

As an urgent medical crisis, antimicrobial resistance has become one of the biggest threats to public health worldwide in the last few decades, mainly due to the inappropriate use of antibiotics. The emergence of carbapenem-resistant gram-negative pathogens, especially carbapenem-resistant Klebsiella pneumoniae (CRKP), poses a difficult clinical therapeutic challenge since carbapenems have been regarded as highly effective antimicrobial agents for treating severe multidrug-resistant bacterial infections $[1,2]$. CRKP infection is endemic in China with a high probability of occurrence and prevalence, which indicates that improving CRKP infection control and treatment levels is urgent and essential in national medical institutions [3].

In CRKP treatment, combination therapy is considered the standard option for CRKP infection treatment because of its contribution to lower mortality rates, instead of 
monotherapy. Unfortunately, there are only a few available antimicrobial agents for clinical use. Tigecycline, fosfomycin, aztreonam, polymyxins, and aminoglycosides are the mainstays of CRKP treatment. In some cases, high-dose and prolonged infusion of carbapenems or double-carbapenem therapy could also be applied as therapeutic options. Therefore, there is an urgent need to develop effective drugs against CRKP infection $[4,5]$. New antibiotics such as plazomicin, eravacycline, meropenem-vaborbacatam and ceftazidime-avibactam (CAZ-AVI) have been proven to be significant complements for CRKP infection treatment [6].

CAZ-AVI, a novel combination of cephalosporin and $\beta$-lactamase inhibitor, was first approved to treat complicated intra-abdominal infections and complicated urinary tract infections by the U.S. Food and Drug Administration in February 2015 [7]. In China, CAZ-AVI has been of great concern for its confirmed clinical efficacy against CRKP infection by inhibiting the activities of extended-spectrum $\beta$-lactamase (ESBL), AmpC-producing $\beta$-lactamase, Klebsiella pneumoniae carbapenemase (KPC) and OXA-48 carbapenemase since its initial marketing in September 2019 [8-11].

It is acknowledged that the selection of antiCRKP agents depends on infectious severity, patients' clinical information, and most importantly, in vitro susceptibility results [6]. Given the therapeutic difficulty, clinicians usually prescribe CAZ-AVI combined with another susceptible (if any) antimicrobial agent for eradicating CRKP. Although several in vitro studies have shown that using CAZ-AVI combined with an extra anti-CRKP agent was synergistic against CRKP $[12,13]$, no differences in mortality and microbiological cure rates were observed in patients receiving CAZ combination therapy or CAZ monotherapy by both studies of Onorato et al. [14] and Fiore et al. [15]. Moreover, no clinical study has investigated whether using CAZ-AVI combination schemes with an in vitro non-susceptible antimicrobial drug could have better clinical effectiveness than CAZ-AVI monotherapy for treating CRKP infection.
Therefore, this study primarily aims to determine whether CAZ-AVI in combination with an in vitro non-susceptible antibiotic is superior to CAZ-AVI monotherapy against CRKP infection in critically ill patients based on addressing potential indication bias.

\section{METHODS}

\section{Study Design and Participants}

We performed a retrospective cohort study at two tertiary hospitals in Shanghai, China. This study was approved by the Institutional Review Board of Huashan Hospital Affiliated to Fudan University and Ruijin Hospital Affiliated to Shanghai Jiao Tong University School of Medicine. The study was performed according to the ethical standards of the Declaration of Helsinki 1964 and its later amendments or comparable ethical standards. All data in this study were extracted from the electronic medical record information system in each hospital.

All patients aged $\geq 18$ years admitted to the intensive care unit (ICU) from January 2019 to December 2020, who had documented CRKP infections (according to microbiological culture tests) with susceptibility testing results and had received at least one dose of CAZ-AVI infusion for treatment were included in our cohort. The exclusion criteria were as follows: (1) patients who received previous CAZ-AVI treatment before the current study began; (2) patients who received CAZ-AVI treatment for $<72 \mathrm{~h}$ or died within this period; (3) a CAZ-AVI-resistant pathogen was isolated from patients; (4) patients received CAZ-AVI accompanied with a second in vitro susceptible agent as combination therapy, according to the susceptibility testing result; and (5) patients with missing data. Only the first course was considered in our study if patients underwent more than one treatment with CAZ-AVI.

\section{Ceftazidime-Avibactam Dosing Regimen}

The duration of CAZ-AVI treatment was at the discretion of the clinicians. A fixed-dose of CAZ- 
Table 1 CAZ-AVI dose adjustment for patients with moderate or severe renal impairment

\begin{tabular}{llll}
\hline $\begin{array}{l}\text { CrCl }(\mathbf{m l} / \\
\mathbf{m i n})\end{array}$ & $\begin{array}{l}\text { Dose } \\
(\mathrm{g})\end{array}$ & $\begin{array}{l}\text { Dosing Interval } \\
(\mathbf{h})\end{array}$ & $\begin{array}{l}\text { Infusion time } \\
(\mathbf{h})\end{array}$ \\
\hline $31 \sim 50$ & 1.25 & Every 8 & $\geq 2$ \\
$16 \sim 30$ & 0.94 & Every 12 & $\geq 2$ \\
$6 \sim 15$ & 0.94 & Every 24 & $\geq 2$ \\
$\leq 5$ & 0.94 & Every 48 & $\geq 2$ \\
\hline
\end{tabular}

AVI (2.5 g) was administered every $8 \mathrm{~h}$ with a 2-h infusion time. Dose adjustment was applied for patients with moderate or severe renal impairment (creatinine clearance $[\mathrm{CrCl}] \leq 50 \mathrm{~mL} / \mathrm{min})$. The dose adjustment details are presented in Table 1. Patients who underwent any mode of continuous renal replacement therapy (CRRT) received a usual dosage regimen of $2.5 \mathrm{~g}$ q8h (infusion time $\geq 2 \mathrm{~h}$ ) because of limited clinical evidence [16].

\section{Study Objectives, Definitions, and Variables}

The primary outcome of our study was 30-day mortality. Combination therapy was considered prescribing a non-susceptible anti-CRKP agent accompanied with CAZ-AVI within $48 \mathrm{~h}$ of starting CAZ-AVI treatment (defined as the starting time of combination therapy) and maintaining a therapeutic duration of at least $72 \mathrm{~h}$. The 30-day microbiological eradication rate was also calculated to evaluate the clinical efficacy of the CAZ-AVI combination therapy. Microbiological eradication was defined as the disappearance of CRKP in all subsequent cultures.

Variables that were possibly associated with 30-day mortality included age, sex, weight, site of infection (defined in accordance with the Centers for Disease Control and Prevention (CDC) criteria [17]); sepsis when starting CAZAVI therapy (identified by Sequential Organ Failure Assessment (SOFA) score of 2 points or more [18]); polymicrobial infections; Acute
Physiology and Chronic Health Evaluation II (APACHE II) scores at the onset of CAZ-AVI therapy [19]; $\mathrm{CrCl}$ (calculated by CockcroftGault formula [20]) at the beginning of CAZAVI therapy; CRRT within the duration of CAZAVI therapy; length of ICU stay before starting CAZ-AVI therapy; concomitant use of vasoactive drugs and mechanical ventilation with the initiation of CAZ-AVI therapy; Charlson comorbidity index (CCI) score [21] and comorbidities; time to start CAZ-AVI therapy and CAZ-AVI treatment duration.

\section{Microbiology}

All pathogen isolation and antimicrobial susceptibility tests (except CAZ-AVI) were carried out using the Vitek 2 Compact system (bioMérieux, Inc.). The susceptibility of CAZ-AVI was determined by the disk-diffusion method (Kirby-Bauer method). The diameter of inhibition zone for CAZ-AVI $\geq 21 \mathrm{~mm}$ and $\leq 20 \mathrm{~mm}$ represented susceptibility and resistance, respectively. The Clinical and Laboratory Standards Institute (CLSI) criteria 2019 were used as the evaluation standard of breakpoints to interpret all antibiotic susceptibility testing results.

\section{Statistical Analysis}

All statistical analyses were performed using SPSS version 26.0 IBM Corp. (Armonk, NY, USA). Each variable was assessed using bivariate analysis of 30-day mortality. The Shapiro-Wilk test was carried out to verify the normality of the distribution of variables separately. The chisquare test or Fisher's exact test was applied for analyzing categorical variables and calculating $P$-value. In contrast, the Student's $t$-test or Mann-Whitney $U$ test was used to analyze continuous variables and calculate $P$-values. Any variable with a $P$-value $\leq 0.20$ was selected to execute a forward stepwise selection for building a Cox proportional hazards regression model. Covariates with $P$-values $\leq 0.10$ remained in the model.

To adjust for confounding by indication, comparison of variables between Combination 
therapy and monotherapy was performed at first. Variables with $P$-values $\leq 0.20$ were included in the Cox proportional hazards regression model for 30-day mortality, while only those with $P$-values $\leq 0.10$ were maintained in this model. Moreover, a propensity score was calculated by the logistic regression model covering the aforementioned variables with $P$ values $\leq 0.10$ and examined in the final model.

The proportional hazard assumption was assessed graphically by the plot of $\log [-\log (-$ survival)] versus $\log$ (time). The collinearity between covariates was also checked. Tests for interactions were not performed. All tests were two-tailed, and $P$-values $\leq 0.05$ were considered statistically significant.

\section{RESULTS}

From January 2019 to December 2020, a total of 62 eligible patients were included in our study (Fig. 1): 36 patients were hospitalized in Huashan Hospital Affiliated to Fudan University and 26 were in Ruijin Hospital Affiliated to Shanghai Jiao Tong University School of Medicine. The mean age of the 62 patients was $60.9 \pm 17.1$ and their mean weight was

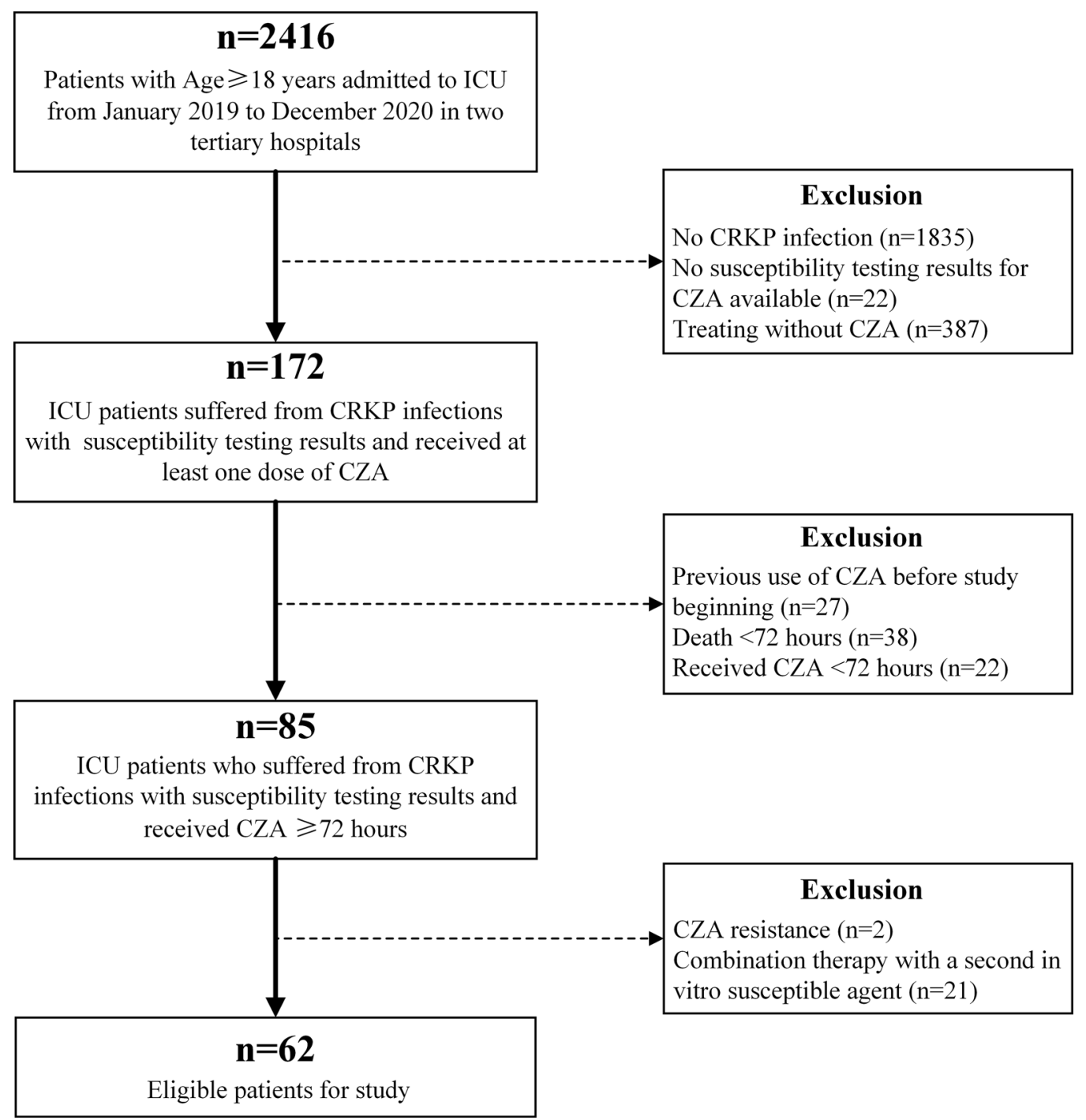

Fig. 1 Study design 
$66.3 \pm 13.5 \mathrm{~kg}$. Forty-seven patients $(75.8 \%)$ were male. As for the primary infection site, 25 patients $(40.3 \%)$ were identified as respiratory infection, as well as nine for bloodstream infection (14.5\%), 12 for abdominal infection (19.4\%), 11 for urinary tract infection $(17.7 \%)$ and 5 for other infections (8.1\%). In addition, $40(64.5 \%)$ and $12(19.4 \%)$ patients had sepsis and polymicrobial infections, respectively. The median APACHE II score at the onset of CAZAVI therapy was 17.5 (interquartile range [IQR], 14.8-20).

According to the CAZ-AVI dosing regimen, 12 patients (19.4\%) received dose adjustment when starting CAZ-AVI therapy because of their lower $\mathrm{CrCl}$ level without CRRT treatment. The median average CAZ-AVI treatment duration was 14 days (IQR, 10-14 days).

The overall 30-day mortality was 33.9\% (21 patients), and the median time of death was 14 days (IQR, 9.5-22.5). There were 41 (66.1\%) patients who received CZA-AVI combination therapy (25 in Huashan Hospital Affiliated to Fudan University and 16 in Ruijin Hospital Affiliated to Shanghai Jiao Tong University School of Medicine) and 21 (33.9\%) with CZAAVI monotherapy (11 in Huashan Hospital Affiliated to Fudan University and 10 in Ruijin Hospital Affiliated to Shanghai Jiao Tong University School of Medicine).

The 30-day microbiological eradication rate was $54.8 \%$ (34 patients). There were 25 patients (61.0\%) in the combination therapy group and nine in the monotherapy group (42.9\%).

As for the starting time for combination therapy, 37 patients (90.2\%) received CAZ-AVI and another non-susceptible antimicrobial at the meantime, while three $(7.3 \%)$ patients and one $(2.4 \%)$ patient were prescribed another non-susceptible antimicrobial at 24 and $48 \mathrm{~h}$ after administering the first dose of CAZ-AVI, respectively. The dosing regimens for the combined antimicrobial agents are described in Table 2. The 30-day mortality rates for patients in the combination therapy and monotherapy groups were $24.4 \%(10 / 41)$ and $47.6 \%(11 / 21)$, respectively $(P=0.028)$. The mortality rates for patients receiving combination therapy and monotherapy were $9.3 / 1000$ patient days and
Table 2 Concomitant antimicrobial agents in CAZ-AVI combination therapy scheme

\begin{tabular}{|c|c|c|c|}
\hline $\begin{array}{l}\text { Antimicrobial } \\
\text { agents }\end{array}$ & $n=41$ & $\begin{array}{l}\text { Dose } \\
\text { regimen }\end{array}$ & $\begin{array}{l}\text { 30-day } \\
\text { mortality } \\
n=10 \\
(24.4 \%)\end{array}$ \\
\hline Meropenem & $11(26.8)$ & $\begin{array}{l}1 \text { for } 500 \mathrm{mg} \\
\mathrm{qd} \\
1 \text { for } 500 \mathrm{mg} \\
\mathrm{q} 12 \\
1 \text { for } 1000 \mathrm{mg} \\
\mathrm{q} 12 \mathrm{~h} \\
8 \text { for } 1000 \mathrm{mg} \\
\mathrm{q} 8 \mathrm{~h}\end{array}$ & $3(27.3)$ \\
\hline Imipenem & $3(7.3)$ & $\begin{array}{l}2 \text { for } 1000 \mathrm{mg} \\
\mathrm{q} 8 \mathrm{~h} \\
1 \mathrm{for} 1000 \mathrm{mg} \\
\mathrm{q} 6 \mathrm{~h}\end{array}$ & $1(33.3)$ \\
\hline Tigecycline $^{\mathrm{a}}$ & $9(22.0)$ & $\begin{array}{c}9 \text { for } 50 \mathrm{mg} \\
\mathrm{q} 12 \mathrm{~h}\end{array}$ & $3(33.3)$ \\
\hline Amikacin & $10(24.4)$ & $\begin{array}{l}1 \text { for } 600 \mathrm{mg} \\
\mathrm{qd} \\
3 \mathrm{for} 800 \mathrm{mg} \\
\mathrm{qd} \\
4 \mathrm{for} 1000 \mathrm{mg} \\
\mathrm{qd} \\
1 \mathrm{for} 1200 \mathrm{mg} \\
\mathrm{qd} \\
1 \text { for } 1400 \mathrm{mg} \\
\mathrm{qd}\end{array}$ & $1(10)$ \\
\hline Fosfomycin & $6(14.6)$ & $\begin{array}{l}2 \text { for } 4 \mathrm{~g} \mathrm{q} 8 \mathrm{~h} \\
4 \text { for } 4 \mathrm{~g} \mathrm{q} 6 \mathrm{~h}\end{array}$ & $2(33.3)$ \\
\hline Aztreonam & $2(4.9)$ & 2 for $2 \mathrm{~g} \mathrm{q} 8 \mathrm{~h}$ & 0 \\
\hline
\end{tabular}

${ }^{a}$ All 9 patients were given first dose tigecycline $100 \mathrm{mg}$ as loading dose

24.9/1000 patient days, $P=0.014$ (log-rank, Fig. 2), respectively. 


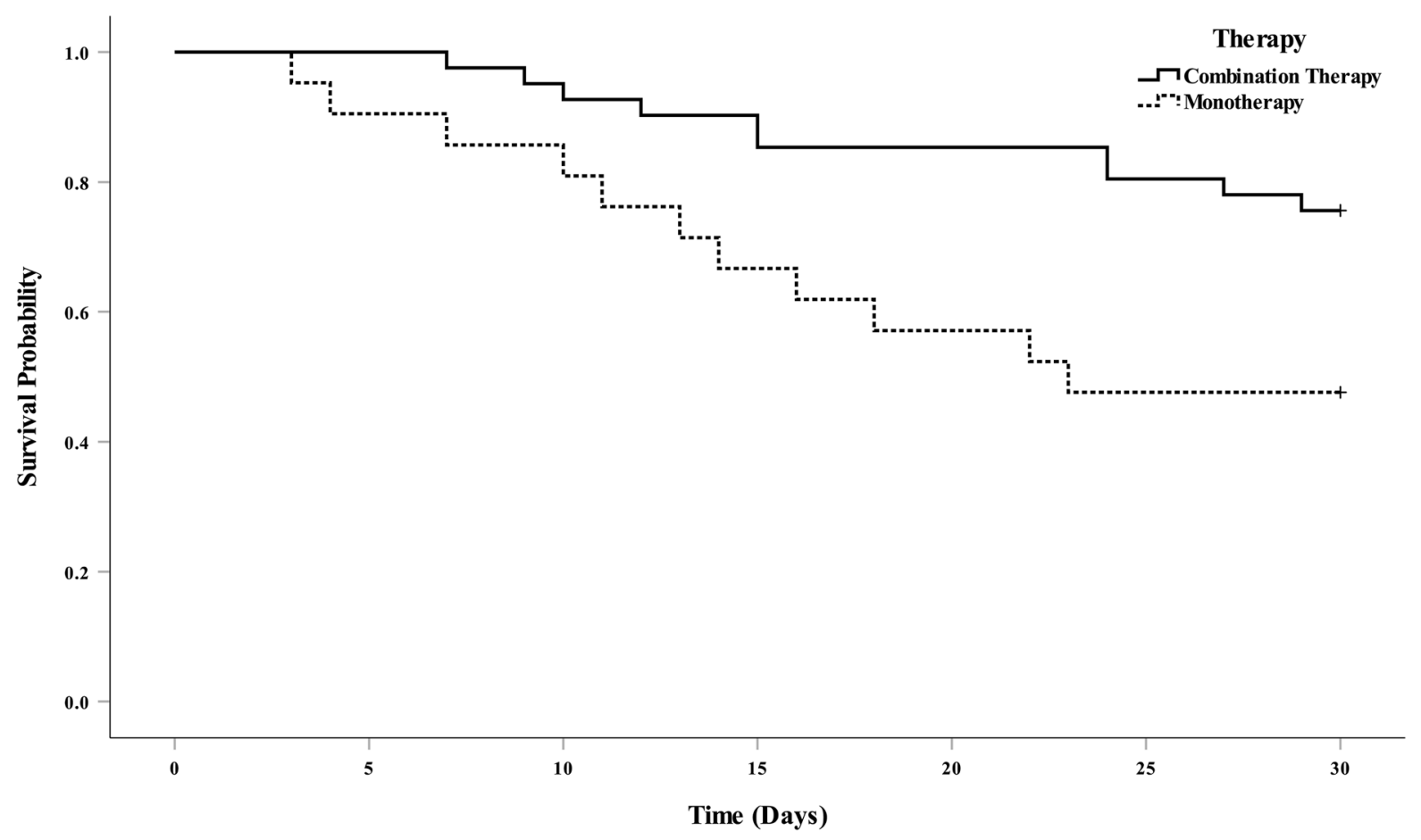

Fig. 2 Survival curves of critically ill patients with CAZAVI combination therapy (CAZ-AVI and another in vitro non-susceptible antimicrobial) (solid line) and CAZ-AVI monotherapy (dashed line) for treating CRKP infection. The mortality rates were $9.3 / 1000$ patient days in

Table 3 displays the details of patient characteristics in the combination and monotherapy groups. Age $(P=0.079)$, respiratory infection $(P=0.166)$, sepsis $(P=0.169)$, length of ICU stay before starting CAZ-AVI therapy $(P=0.059)$ and CCI score $(P=0.090)$ were chosen for stepwise variable selection in the Cox proportional hazards regression model and creation of the propensity score.

Table 4 shows the bivariate analysis results for the 30-day mortality. Other infections $(P=0.157)$, polymicrobial infection $(P=0.195)$, APACHE II score at the onset of CAZ-AVI therapy $(P=0.032), \mathrm{CrCl}(P=0.076)$, vasoactive drugs $(P<0.001)$, mechanical ventilation $(P=0.008)$, cardiovascular disease $(P=0.028)$, respiratory disease $(P=0.082)$, liver disease $(P=0.111)$, organ transplantation $(P<0.001)$, neoplasia $(P=0.111)$ and combination therapy $(P=0.028)$ were also included in the Cox regression model for stepwise variable selection. combination therapy group and 24.9/1000 patient days in monotherapy group, $P=0.014$

The multivariate analysis results are listed in Table 5. Combination therapy was significantly associated with lower 30-day mortality (Hazard ratio, $0.167 ; 95 \%$ Confidence Interval, $0.060-0.465 ; P=0.001)$. In contrast, a higher APACHE II score at the onset of CAZ-AVI therapy, vasoactive drugs, and comorbidity of organ transplantation were considered factors that increased 30-day mortality. Moreover, the propensity score showed no significant alteration with the results of the other variables in the Cox regression model.

\section{Subgroup Analysis}

CAZ-AVI combination therapy could be conducive to lower 30-day mortality when patients received carbapenems, tigecycline and fosfomycin as another concomitant agent, compared with CAZ-AVI monotherapy. Furthermore, combination therapy was a 
Table 3 Characteristics of patients receiving CAZ-AVI combination and monotherapy antimicrobial treatment

\begin{tabular}{|c|c|c|c|}
\hline Variable $^{a}$ & Combination $(n=41)$ & $\operatorname{Monotherapy}(n=21)$ & $P$-value \\
\hline Age, years & $58.2 \pm 18.4$ & $66.2 \pm 13.2$ & 0.079 \\
\hline Sex (male) & $33(80.5)$ & $14(66.7)$ & 0.229 \\
\hline Weight, kg & $67.8 \pm 14.2$ & $63.2 \pm 11.6$ & 0.207 \\
\hline \multicolumn{4}{|l|}{ Primary site of infection } \\
\hline Primary bloodstream infection & $7(17.1)$ & $2(9.5)$ & 0.705 \\
\hline Respiratory infection & $14(34.1)$ & $11(52.4)$ & 0.166 \\
\hline Abdominal infection & $9(22.0)$ & $3(14.3)$ & 0.735 \\
\hline Urinary tract infection & $7(17.1)$ & $4(19.0)$ & 1.000 \\
\hline Other infections & $4(9.8)$ & $1(4.8)$ & 0.654 \\
\hline Sepsis & $24(58.5)$ & $16(76.2)$ & 0.169 \\
\hline Polymicrobial infection & $9(22.0)$ & $3(14.3)$ & 0.735 \\
\hline APACHE II score (CAZ-AVI onset) & $18(14-20.5)$ & $17(16-19)$ & 0.846 \\
\hline $\mathrm{CrCl}, \mathrm{mL} / \mathrm{min}$ & $76.7(42.5-133.6)$ & $97.5(60.0-131.9)$ & 0.409 \\
\hline CRRT & $4(9.8)$ & $2(9.5)$ & 1.000 \\
\hline Length of ICU stay before starting CAZ-AVI therapy, days & $17(8-31)$ & $32(9.5-58.5)$ & 0.059 \\
\hline Vasoactive drugs & $24(58.5)$ & $13(61.9)$ & 0.798 \\
\hline Mechanical ventilation & $26(63.4)$ & $13(61.9)$ & 0.907 \\
\hline \multicolumn{4}{|l|}{ Comorbidities } \\
\hline Cardiovascular disease & $10(24.4)$ & $6(28.6)$ & 0.722 \\
\hline Respiratory Disease & $18(43.9)$ & $8(38.1)$ & 0.661 \\
\hline Central Nervous system disease & $7(17.1)$ & $4(19.0)$ & 1.000 \\
\hline Autoimmune disease & $4(9.8)$ & $2(9.5)$ & 1.000 \\
\hline Liver disease & $13(31.7)$ & $7(33.3)$ & 0.897 \\
\hline Renal insufficiency & $11(26.8)$ & $4(19.0)$ & 0.498 \\
\hline Diabetes & $8(19.5)$ & $7(33.3)$ & 0.229 \\
\hline Organ transplantation & $6(14.6)$ & $4(19.0)$ & 0.722 \\
\hline Neoplasia & $13(31.7)$ & $7(33.3)$ & 0.897 \\
\hline CCI score & $4(3-5)$ & $4(3.5-6)$ & 0.090 \\
\hline CAZ-AVI treatment duration, days & $14(12-14)$ & $14(10-14)$ & 0.299 \\
\hline
\end{tabular}

All data are exhibited as number (\%), mean $\pm \mathrm{SD}$ or median $\left(\mathrm{P}_{25}-\mathrm{P}_{75}\right)$ 
Table 4 Potential risk factors for 30-day mortality in patients treated with CAZ-AVI

\begin{tabular}{|c|c|c|c|}
\hline \multirow[t]{2}{*}{ Variable $^{\mathrm{a}}$} & \multicolumn{2}{|l|}{ 30-day Mortality } & \multirow[t]{2}{*}{$P$-value } \\
\hline & Survival $(n=41)$ & Death $(n=21)$ & \\
\hline Age, years & $57.9 \pm 17.2$ & $66.7 \pm 15.8$ & 0.055 \\
\hline Sex (male) & $31(75.6)$ & $16(76.2)$ & 0.960 \\
\hline Weight, kg & $66.2 \pm 13.8$ & $66.4 \pm 13.1$ & 0.949 \\
\hline \multicolumn{4}{|l|}{ Primary site of infection } \\
\hline Primary bloodstream infection & $7(17.1)$ & $2(9.5)$ & 0.705 \\
\hline Respiratory infection & $13(31.7)$ & $12(57.1)$ & 0.053 \\
\hline Abdominal infection & $7(17.1)$ & $5(23.8)$ & 0.520 \\
\hline Urinary tract infection & $9(22.0)$ & $2(9.5)$ & 0.305 \\
\hline Other infections & $5(12.2)$ & 0 & 0.157 \\
\hline Sepsis & $22(53.7)$ & $18(85.7)$ & 0.014 \\
\hline Polymicrobial infection & $10(24.4)$ & $2(9.5)$ & 0.195 \\
\hline APACHE II score (CAZ-AVI onset) & $16(14-19.5)$ & $18(17-21)$ & 0.032 \\
\hline $\mathrm{CrCl}, \mathrm{mL} / \mathrm{min}$ & $100.2(55.3-142.0)$ & $61.4(33.8-108.7)$ & 0.076 \\
\hline CRRT & $3(7.3)$ & $3(14.3)$ & 0.398 \\
\hline Length of ICU stay before starting CAZ-AVI therapy, days & $21(7.5-33.5)$ & $23(13-51.5)$ & 0.198 \\
\hline Vasoactive drug & $17(41.5)$ & $20(95.2)$ & $<0.001$ \\
\hline Mechanical ventilation & $21(51.2)$ & $18(85.7)$ & 0.008 \\
\hline \multicolumn{4}{|l|}{ Comorbidities } \\
\hline Cardiovascular disease & $7(17.1)$ & $9(42.9)$ & 0.028 \\
\hline Respiratory disease & $14(34.1)$ & $12(57.1)$ & 0.082 \\
\hline Central Nervous system disease & $8(19.5)$ & $3(14.3)$ & 0.735 \\
\hline Autoimmune disease & $5(12.2)$ & $1(4.8)$ & 0.654 \\
\hline Liver disease & $16(39.0)$ & $4(19.0)$ & 0.111 \\
\hline Renal insufficiency & $9(22.0)$ & $6(28.6)$ & 0.565 \\
\hline Diabetes & $9(22.0)$ & $6(28.6)$ & 0.565 \\
\hline Organ transplantation & $1(2.4)$ & $9(42.9)$ & $<0.001$ \\
\hline Neoplasia & $16(39.0)$ & $4(19.0)$ & 0.111 \\
\hline CCI score & $4(3-5)$ & $4(3.5-5)$ & 0.311 \\
\hline Combination therapy & $31(75.6)$ & $10(47.6)$ & 0.028 \\
\hline CAZ-AVI treatment duration, days & $14(12-14)$ & $14(9.5-18)$ & 0.704 \\
\hline
\end{tabular}

All data are exhibited as number $(\%)$, mean $\pm \mathrm{SD}$ or median $\left(\mathrm{P}_{25}-\mathrm{P}_{75}\right)$ 
Table 5 Cox-proportional hazards regression model for 30-day mortality ${ }^{a}$

\begin{tabular}{lrll}
\hline Variable $^{\mathbf{b}}$ & HR & 95\% CI & $\begin{array}{l}\boldsymbol{P} \text { - } \\
\text { value }\end{array}$ \\
\hline Combination therapy & 0.167 & $0.060-0.465$ & 0.001 \\
$\begin{array}{l}\text { APACHE II score } \\
\quad \text { CAZ-AVI onset) }\end{array}$ & 1.180 & $1.027-1.356$ & 0.019 \\
$\quad$ & & \\
Vasoactive drugs & 14.732 & $1.881-115.407$ & 0.010 \\
Organ transplantation & 3.817 & $1.475-9.881$ & 0.006 \\
\hline
\end{tabular}

${ }^{a} \mathrm{~A}$ propensity score for prescribing combination therapy included age, respiratory infection, sepsis, length of ICU stay before starting CAZ-AVI therapy and CCI score in a logistic regression model using a Likelihood Ratio (LR) forward stepwise method (Hosmer-Lemeshow goodnessof-fit test: $\chi^{2}$-square $\left.=7.478 ; P=0.486\right)$. The propensity score that was included in the final Cox-proportional hazards regression model showed no significant alteration with the results of other variables $(P=0.926)$

${ }^{\mathrm{b}}$ Age, sepsis, respiratory infection, length of ICU stay before starting CAZ-AVI therapy, CCI score in Table 1 and other infections, polymicrobial infection, $\mathrm{CrCl}$, mechanical ventilation, cardiovascular disease, respiratory Disease, liver disease, neoplasia in Table 2 were all checked but excluded finally in the Cox-proportional hazards regression model because the $P$-value $>0.10$ for each aforementioned variable

${ }^{\mathrm{c}} H R$ Hazard ratio

${ }^{\mathrm{d}} C I$ confidence interval

protective factor in the subgroup of patients with sepsis or $\mathrm{CrCl}>50 \mathrm{~mL} / \mathrm{min}$. Patients who stayed in ICU for $\leq 30$ days or underwent mechanical ventilation when starting CAZ-AVI therapy also benefited from CAZ-AVI combination therapy for decreased mortality as well (Table 6).

\section{DISCUSSION}

As the first novel antibiotic coming to the market against CRKP, CZA-AVI is of great concern to clinicians and pharmacists. Although several studies have reported the rapidly developing resistance of CAZ-AVI [22-25], it was still considered a first-line anti-CRKP agent due to its
Table 6 Hazard ratio of CAZ-AVI combination therapy and 30-day mortality according in the subgroup analysis

\begin{tabular}{|c|c|c|c|c|}
\hline Subgroup $^{a}$ & $n$ & $H_{R^{b}}$ & $95 \% \mathrm{CI}^{\mathrm{c}}$ & $\begin{array}{l}P \text { - } \\
\text { value }\end{array}$ \\
\hline $\begin{array}{l}\text { Combination with } \\
\text { carbapenem }^{\mathrm{d}}\end{array}$ & 35 & 0.222 & $0.053-0.938$ & 0.041 \\
\hline $\begin{array}{l}\text { Combination with } \\
\text { tigecycline }\end{array}$ & 30 & 0.220 & $0.052-0.936$ & 0.040 \\
\hline $\begin{array}{l}\text { Combination with } \\
\text { fosfomycin }\end{array}$ & 27 & 0.101 & $0.016-0.638$ & 0.015 \\
\hline Sepsis & 40 & 0.136 & $0.039-0.474$ & 0.002 \\
\hline $\mathrm{CrCl}>50 \mathrm{~mL} / \mathrm{min}$ & 45 & 0.219 & $0.065-0.741$ & 0.015 \\
\hline $\begin{array}{l}\text { ICU stay before } \\
\text { starting CAZ-AVI } \\
\text { therapy } \leq 30 \mathrm{~d}\end{array}$ & 41 & 0.139 & $0.036-0.542$ & 0.004 \\
\hline Mechanical ventilation & 39 & 0.214 & $0.066-0.686$ & 0.010 \\
\hline
\end{tabular}

${ }^{a}$ Adjusted for APACHE II score (CAZ-AVI onset), vasoactive drugs and organ transplantation

${ }^{\mathrm{b}} H R$ Hazard ratio

${ }^{\mathrm{c}} \mathrm{CI}$ confidence interval

${ }^{\mathrm{d}}$ Eleven patients received meropenem and three patients received imipenem

superiority to the current polymyxin-based therapy efficacy and safety $[26,27]$. However, it remains unclear whether CAZ-AVI should be used as monotherapy or in combination with other agents in the article by Jason et al. [28] Therefore, we performed a study to compare with CAZ-AVI combination therapy and CAZAVI monotherapy for patients with CRKP infections for the first time.

Our study found that CAZ-AVI, combined with another in vitro non-susceptible antimicrobial, could significantly lower the 30-day mortality of patients with CRKP infection. A higher microbiological eradication rate was observed in patients receiving CAZ-AVI combination therapy than those who received CAZAVI monotherapy. This was an important conclusion because the CAZ-AVI combination therapy revealed sufficient clinical efficacy, given the developing resistance of few therapeutic drugs against CRKP. In addition, the 
therapeutic effect of the CAZ-AVI combination therapy did not depend on the susceptibility to other antimicrobials. This might refer to conventional antimicrobial susceptibility test methods such as disk diffusion, dilution, E-test, and even automated systems, could not predict clinical efficacy for infectious treatment in vivo precisely because they failed to simulate most aspects of host environments in bacteriological media $[29,30]$. David et al. showed that carbapenem resistance in Enterobacteriaceae could be falsely detected by utilizing antimicrobial susceptibility testing methods due to different zinc concentrations in conventional broth and at infection sites [31, 32]. These results imply that some carbapenem-resistant organisms tested by antimicrobial susceptibility tests in vitro might be susceptible to in vivo, providing good support for our conclusions.

Furthermore, carbapenems, tigecycline, and fosfomycin were recognized as effective concomitant agents to decrease 30-day mortality by subgroup analysis. This indicates that CAZ-AVI in combination with fosfomycin could be a potential therapeutic strategy to treat CRKP infection, which is consistent with the conclusion of Ojdana et al.'s in vitro study that CAZAVI with fosfomycin could enhance antibacterial activity against carbapenemase-producing Klebsiella pneumonia [13].

As another combined drug with CZA-AVI, tigecycline was proved clinically effective on patients with CRKP infection in our study. At the same time, unsatisfactory results were described by Ojdana et al. [13] and Gaibani et al. [33], who reported that only $5 \%$ and $8 \%$ isolates of carbapenemase-producing Klebsiella pneumoniae received the synergistic effect of these two agents, respectively. Nevertheless, these two aforementioned studies were both in vitro and lacked clinical isolates to verify the credibility of their conclusions. In addition, a clinical case of CZA-AVI plus tigecycline successfully treated a 61-year-old man with intra-abdominal carbapenemase-producing Klebsiella pneumoniae infection [34]. We believed that CAZ-AVI plus tigecycline might be another meaningful therapeutic combination against CRKP based on the above reasons.
We also found that carbapenems (meropenem and imipenem) played a crucial role in combination therapy with CZA-AVI as a protective factor against 30-day mortality. Ertapenem was not included in our study because it was only applied to dual carbapenem treatment as an anti-CRKP combination partner [35]. Gaibani et al. [33] suggested that the combination of CAZ-AVI and imipenem could be a therapeutic option against CRKP. Mikhail et al. evaluated the synergistic activity of CZA-AVI and other antimicrobials. Their data revealed that CZA-AVI combined with meropenem had the potential synergy to treat multidrug-resistant Klebsiella pneumoniae and Pseudomonas aeruginosa [12]. Although this treatment scheme is rare in clinical practice because CZAAVI and carbapenems are both $\beta$-lactam antibiotics, it might be similar to the efficacy of double carbapenem therapy as a rescue strategy for the treatment of CRKP [36-38]. Further investigation should be carried out to determine the possible mechanisms of increasing anti-CRKP activity between CZA-AVI with carbapenems and which carbapenem was the optimum choice in clinical practice, as well as the appropriate dose regimen.

Moreover, we could not neglect two other types of anti-CRKP agents, namely aztreonam and polymyxins (polymyxin $\mathrm{B}$ and colistin). The combination of CAZ-AVI and aztreonam has already been reported as a promising treatment option against carbapenemase-producing pathogens, especially metallo-beta-lactamaseproducing gram-negative bacteria [39-42]. Polymyxins (polymyxin B and colistin) were addressed as the last resort antibiotics to treat CRKP infection before CAZ-AVI in clinical practice [1, 43-45]. However, a combination of CAZ-AVI and polymyxins has not been found in any clinical study. In our study, aztreonam and polymyxins were not evaluated as in vitro nonsusceptible antibiotics with CAZ-AVI because aztreonam resistance was only emerged in five patients who received CAZ-AVI for less than $72 \mathrm{~h}$, and no isolate was found to be resistant to polymyxin B and colistin in our study. These two agents should be included in further studies. 
In the current study, CZA-AVI combination therapy was also beneficial to patients with sepsis or those receiving mechanical ventilation, which implied that combination therapy was reasonable for treating critically ill patients with CRKP infection, especially for patients who stayed in the ICU for less than 30 days when starting using CZA-AVI. Combination therapy also showed a protective effect on mortality in patients with $\mathrm{CrCl}>50 \mathrm{~mL} / \mathrm{min}$, who received a non-adjusted dose of CAZ-AVI during the treatment duration. It might be concluded that a higher dose of CAZ-AVI could effectively lower mortality. However, the current CAZ-AVI dosage regimen has been verified by population pharmacokinetic models in which a high probability of target attainment (>95\%) was observed in patients with various $\mathrm{CrCl}$ levels, except for patients with $\mathrm{CrCl}$ between 8 and $15 \mathrm{~mL} / \mathrm{min}$, according to the research by Das et al. [46]. Hence, we maintained that the lower mortality could not simply be attributed to the use of non-adjusted dose of CAZ-AVI. Renal insufficiency should be considered a rational factor for poor outcomes in critically ill patients [47].

We attempted to control the potential for confounding bias by indication in this study. We used a multivariate model to evaluate all the possible associated variables with combination therapy by the forward stepwise selection and included the propensity scores which creating by these same variables. Although age, sepsis, respiratory infection, length of ICU stay before starting CAZ-AVI therapy, and CCI score were included for evaluation in the multivariate model, none of these variables remained in the final model. In addition, the propensity score was included without any significant alteration with other variables in the final Cox proportional hazards regression model. Consequently, we believe that the indication bias could barely affect our study results.

The current study had some limitations. First, it was a retrospective cohort study with a small sample size. Well-designed prospective studies or randomized control trials with more participants should be designed for further investigation. Second, carbapenemase detection tests such as the Carba NP (CNP) test or modified carbapenem inactivation method (mCIM), were not performed in our study due to the lack of necessary devices and reagents in the clinical laboratory. Last but not least, only dual agents' combination was evaluated in our study. Three or more drug combination schemes should be investigated in future studies.

\section{CONCLUSIONS}

In conclusion, our study showed that CZA-AVI combined with another in vitro non-susceptible antimicrobial, especially carbapenems, fosfomycin, and tigecycline, could significantly lower the mortality risk in critically ill patients with CRKP infection. Further well-designed prospective studies should be performed to verify if CZA-AVI combination therapy could benefit all patients with CRKP infection and determine the optimum CZA-AVI combination scheme.

\section{ACKNOWLEDGEMENTS}

Funding. This study and the Rapid Service Fee was funded by Shanghai "Rising Stars of Medical Talent" Youth Development ProgramYouth Medical Talents-Clinical Pharmacist Program [SHWRS(2020)_120].

Authorship. All named authors meet the International Committee of Medical Journal Editors (ICMJE) criteria for authorship for this article, take responsibility for the integrity of the work as a whole, and have given their approval for this version to be published.

Authors' Contributions. Guanhao Zheng, Juan He, Liang Zhang, Zhitao Yang and Xiaolan Bian conceived and designed this study. Guanhao Zheng, Jianxin Zhang, Bei Wang, Jiaqi Cai, Lili Wang, Kaixuan Hou and Yan Zhang collected the information in the case, and contributed to the acquisition, analysis, and interpretation of the data. Guanhao Zheng, Jianxin Zhang, Bei Wang, Liang Zhang and Juan 
He wrote and revised the manuscript. All authors read and approved the final manuscript.

Disclosures. Guanhao Zheng, Jianxin Zhang, Bei Wang, Jiaqi Cai, Lili Wang, Kaixuan Hou, Yan Zhang, Liang Zhang, Zhitao Yang, Juan He, Xiaolan Bian have nothing to disclose.

Compliance with Ethics Guidelines. This study was approved by Huashan Hospital Affiliated to Fudan University and Ruijin Hospital Affiliated to Shanghai Jiao Tong University School of Medicine and has been performed in accordance with the ethical standards laid down in "Declaration of Helsinki 1964" and its later amendments or comparable ethical standards.

Data Availability. All data generated or analyzed during this study are included in this published article.

Open Access. This article is licensed under a Creative Commons Attribution-NonCommercial 4.0 International License, which permits any non-commercial use, sharing, adaptation, distribution and reproduction in any medium or format, as long as you give appropriate credit to the original author(s) and the source, provide a link to the Creative Commons licence, and indicate if changes were made. The images or other third party material in this article are included in the article's Creative Commons licence, unless indicated otherwise in a credit line to the material. If material is not included in the article's Creative Commons licence and your intended use is not permitted by statutory regulation or exceeds the permitted use, you will need to obtain permission directly from the copyright holder. To view a copy of this licence, visit http://creativecommons.org/licenses/by$\mathrm{nc} / 4.0 /$.

\section{REFERENCES}

1. Doi Y. Treatment options for carbapenem-resistant gram-negative bacterial infections. Clin Infect Dis. 2019;69(Suppl 7):S565-75.
2. Karam G, Chastre J, Wilcox MH, Vincent JL. Antibiotic strategies in the era of multidrug resistance. Crit Care (London, England). 2016;20(1): 136.

3. Zhang Y, Wang Q, Yin Y, Chen H, Jin L, Gu B, et al. Epidemiology of carbapenem-resistant enterobacteriaceae infections: report from the china CRE network. Antimicrob Agents Chemother. 2018. https://doi.org/10.1128/AAC.01882-17.

4. Morrill HJ, Pogue JM, Kaye KS, LaPlante KL. Treatment options for carbapenem-resistant Enterobacteriaceae infections. Open Forum Infect Dis. 2015;2(2):ofv050.

5. Trecarichi EM, Tumbarello M. Therapeutic options for carbapenem-resistant Enterobacteriaceae infections. Virulence. 2017;8(4):470-84.

6. Sheu CC, Chang YT, Lin SY, Chen YH, Hsueh PR. Infections caused by carbapenem-resistant Enterobacteriaceae: an update on therapeutic options. Front Microbiol. 2019;10:80.

7. Kaye KS, Pogue JM. Infections caused by resistant gram-negative bacteria: epidemiology and management. Pharmacotherapy. 2015;35(10):949-62.

8. Shirley M. Ceftazidime-avibactam: a review in the treatment of serious gram-negative bacterial infections. Drugs. 2018;78(6):675-92.

9. Zhanel GG, Lawson CD, Adam H, Schweizer F, Zelenitsky S, Lagacé-Wiens PR, et al. Ceftazidimeavibactam: a novel cephalosporin/ $\beta$-lactamase inhibitor combination. Drugs. 2013;73(2):159-77.

10. Zasowski EJ, Rybak JM, Rybak MJ. The $\beta$-lactams strike back: ceftazidime-avibactam. Pharmacotherapy. $2015 ; 35(8): 755-70$.

11. Kuang H, Zhong C, Wang Y, Ye H, Ao K, Zong Z, et al. Clinical characteristics and outcomes of patients with multidrug-resistant Gram-negative bacterial infections treated with ceftazidime/avibactam. J Global Antimicrob Resist. 2020;23: 404-7.

12. Mikhail S, Singh NB, Kebriaei R, Rice SA, Stamper $\mathrm{KC}$, Castanheira M, et al. Evaluation of the synergy of ceftazidime-avibactam in combination with meropenem, amikacin, aztreonam, colistin, or fosfomycin against well-characterized multidrug-resistant Klebsiella pneumoniae and Pseudomonas aeruginosa. Antimicrob Agents Chemother. 2019. https://doi.org/10.1128/AAC.00779-19.

13. Ojdana D, Gutowska A, Sacha P, Majewski P, Wieczorek P, Tryniszewska E. Activity of ceftazidime-avibactam alone and in combination with ertapenem, fosfomycin, and tigecycline against 
carbapenemase-producing Klebsiella pneumoniae. Microb Drug Resist (Larchmont, NY). 2019;25(9): 1357-64.

14. Onorato L, Di Caprio G, Signoriello S, Coppola N. Efficacy of ceftazidime/avibactam in monotherapy or combination therapy against carbapenem-resistant Gram-negative bacteria: a meta-analysis. Int J Antimicrob Agents. 2019;54(6):735-40.

15. Fiore M, Alfieri A, Di Franco S, Pace MC, Simeon V, Ingoglia $\mathrm{G}$, et al. Ceftazidime-avibactam combination therapy compared to ceftazidime-avibactam monotherapy for the treatment of severe infections due to carbapenem-resistant pathogens: a systematic review and network meta-analysis. Antibiotics (Basel, Switzerland). 2020;9(7):388.

16. Li L, Li X, Xia Y, Chu Y, Zhong H, Li J, et al. Recommendation of antimicrobial dosing optimization during continuous renal replacement therapy. Front Pharmacol. 2020;11:786.

17. Horan TC, Andrus M, Dudeck MA. CDC/NHSN surveillance definition of health care-associated infection and criteria for specific types of infections in the acute care setting. Am J Infect Control. 2008;36(5):309-32.

18. Singer M, Deutschman CS, Seymour CW, ShankarHari M, Annane D, Bauer M, et al. The third international consensus definitions for sepsis and septic shock (Sepsis-3). JAMA. 2016;315(8):801-10.

19. Knaus WA, Draper EA, Wagner DP, Zimmerman JE. APACHE II: a severity of disease classification system. Crit Care Med. 1985;13(10):818-29.

20. Cockcroft DW, Gault MH. Prediction of creatinine clearance from serum creatinine. Nephron. $1976 ; 16(1): 31-41$.

21. Charlson ME, Pompei P, Ales KL, MacKenzie CR. A new method of classifying prognostic comorbidity in longitudinal studies: development and validation. J Chronic Dis. 1987;40(5):373-83.

22. Wang Y, Wang J, Wang R, Cai Y. Resistance to ceftazidime-avibactam and underlying mechanisms. J Global Antimicrob Resist. 2020;22:18-27.

23. Göttig S, Frank D, Mungo E, Nolte A, Hogardt M, Besier S, et al. Emergence of ceftazidime/avibactam resistance in KPC-3-producing Klebsiella pneumoniae in vivo. J Antimicrob Chemother. 2019;74(11): 3211-6.

24. Shields RK, Potoski BA, Haidar G, Hao B, Doi Y, Chen L, et al. Clinical outcomes, drug toxicity, and emergence of ceftazidime-avibactam resistance among patients treated for carbapenem-resistant enterobacteriaceae infections. Clin Infect Dis. 2016;63(12):1615-8.

25. Giddins MJ, Macesic N, Annavajhala MK, Stump S, Khan S, McConville TH, et al. Successive emergence of ceftazidime-avibactam resistance through distinct genomic adaptations in bla(KPC-2)-harboring klebsiella pneumoniae sequence type 307 isolates. Antimicrob Agents Chemother. 2018. https://doi. org/10.1128/AAC.02101-17.

26. Shields RK, Nguyen MH, Chen L, Press EG, Potoski $\mathrm{BA}$, Marini RV, et al. Ceftazidime-avibactam is superior to other treatment regimens against carbapenem-resistant klebsiella pneumoniae bacteremia. Antimicrob Agents Chemother. 2017. https://doi.org/10.1128/AAC.00883-17.

27. van Duin D, Lok JJ, Earley M, Cober E, Richter SS, Perez F, et al. Colistin versus ceftazidime-avibactam in the treatment of infections due to carbapenemresistant Enterobacteriaceae. Clin Infect Dis. 2018;66(2):163-71.

28. Pogue JM, Bonomo RA, Kaye KS. Ceftazidime/avibactam, meropenem/vaborbactam, or both? Clinical and formulary considerations. Clin Infect Dis. 2019;68(3):519-24.

29. Ersoy SC, Heithoff DM, Barnes L, Tripp GK, House JK, Marth JD, et al. Correcting a fundamental flaw in the paradigm for antimicrobial susceptibility testing. EBioMedicine. 2017;20:173-81.

30. Khan ZA, Siddiqui MF, Park S. Current and emerging methods of antibiotic susceptibility testing. Diagnostics (Basel, Switzerland). 2019;9(2):49.

31. Asempa TE, Abdelraouf K, Nicolau DP. Metallo- $\beta$ lactamase resistance in Enterobacteriaceae is an artefact of currently utilized antimicrobial susceptibility testing methods. J Antimicrob Chemother. 2020;75(4):997-1005.

32. Abdelraouf K, Reyes S, Nicolau DP. The paradoxical in vivo activity of $\beta$-lactams against metallo- $\beta$-lactamase-producing Enterobacterales is not restricted to carbapenems. J Antimicrob Chemother. 2021;76(3):684-91.

33. Gaibani P, Lewis RE, Volpe SL, Giannella M, Campoli $\mathrm{C}$, Landini $\mathrm{MP}$, et al. In vitro interaction of ceftazidime-avibactam in combination with different antimicrobials against KPC-producing Klebsiella pneumoniae clinical isolates. Int J Infect Dis. 2017;65:1-3.

34. Guedes M, Duro R, Fonseca T, Abreu I, Rocha-Pereira N. Carbapenemase-producing Klebsiella pneumoniae intra-abdominal infection successfully treated with ceftazidime/avibactam plus tigecycline. IDCases. 2020;20:e00745. 
35. Fritzenwanker $M$, Imirzalioglu $C$, Herold $S$, Wagenlehner FM, Zimmer KP, Chakraborty T. treatment options for carbapenem- resistant gramnegative infections. Deutsches Arzteblatt Int. 2018;115(20-21):345-52.

36. De Pascale G, Martucci G, Montini L, Panarello G, Cutuli SL, Di Carlo D, et al. Double carbapenem as a rescue strategy for the treatment of severe carbapenemase-producing Klebsiella pneumoniae infections: a two-center, matched case-control study. Crit Care. 2017;21(1):173.

37. Bulik CC, Nicolau DP. Double-carbapenem therapy for carbapenemase-producing Klebsiella pneumoniae. Antimicrob Agents Chemother. 2011;55(6): 3002-4.

38. Souli M, Karaiskos I, Masgala A, Galani L, Barmpouti E, Giamarellou H. Double-carbapenem combination as salvage therapy for untreatable infections by KPC-2-producing Klebsiella pneumoniae. Eur J Clin Microbiol Infect Dis. 2017;36(7):1305-15.

39. Lee M, Abbey T, Biagi M, Wenzler E. Activity of aztreonam in combination with ceftazidime-avibactam against serine- and metallo- $\beta$-lactamaseproducing Pseudomonas aeruginosa. Diagn Microb Infect Dis. 2021;99(1):115227.

40. Avery LM, Nicolau DP. Assessing the in vitro activity of ceftazidime/avibactam and aztreonam among carbapenemase-producing Enterobacteriaceae: defining the zone of hope. Int J Antimicrob Agents. 2018;52(5):688-91.

41. Biagi M, Wu T, Lee M, Patel S, Butler D, Wenzler E. Searching for the optimal treatment for metalloand serine- $\beta$-lactamase producing enterobacteriaceae: aztreonam in combination with ceftazidimeavibactam or meropenem-vaborbactam. Antimicrob Agents Chemother. 2019. https://doi.org/10. 1128/AAC.01426-19.
42. Pragasam AK, Veeraraghavan B, Shankar BA, Bakthavatchalam YD, Mathuram A, George B, et al. Will ceftazidime/avibactam plus aztreonam be effective for NDM and OXA-48-Like producing organisms: Lessons learnt from In vitro study. Indian J Med Microbiol. 2019;37(1):34-41.

43. Karaiskos I, Lagou S, Pontikis K, Rapti V, Poulakou G. The, "old" and the "new" antibiotics for MDR gram-negative pathogens: for whom, when, and how. Front Public Health. 2019;7:151.

44. Liang Q, Huang M, Xu Z. Early use of polymyxin B reduces the mortality of carbapenem-resistant Klebsiella pneumoniae bloodstream infection. Braz J Infect Dis. 2019;23(1):60-5.

45. Bandick RG, Mousavi S, Bereswill S, Heimesaat MM. Review of therapeutic options for infections with carbapenem-resistant Klebsiella pneumoniae. Eur J Microbiol Immunol. 2020;10(3):115-24.

46. Das S, Li J, Riccobene T, Carrothers TJ, Newell P, Melnick D, et al. Dose selection and validation for ceftazidime-avibactam in adults with complicated intra-abdominal infections, complicated urinary tract infections, and nosocomial pneumonia. Antimicrob Agents Chemother. 2019. https://doi. org/10.1128/AAC.02187-18.

47. Clermont G, Acker CG, Angus DC, Sirio CA, Pinsky MR, Johnson JP. Renal failure in the ICU: comparison of the impact of acute renal failure and endstage renal disease on ICU outcomes. Kidney Int. 2002;62(3):986-96.

\section{Publisher's Note}

Springer Nature remains neutral with regard to jurisdictional claims in published maps and institutional affiliations. 